Canad. Math. Bull. Vol. 19 (2), 1976

\title{
A NOTE ON $H$-CLOSED EXTENSIONS OF A PRODUCT
}

\author{
BY \\ ANTHONY J. D'ARISTOTLE
}

1. Introduction. Any Hausdorff space $X$ is a dense subspace of an $H$-closed space $\kappa X$, called the Katĕtov extension of $X$, with the property that any $H$-closed extension $Y$ of $X$ is a continuous image of $\kappa X$ under a mapping which leaves $X$ pointwise fixed [8], [10]. In [8], Liu has shown that the extensions $\kappa(X \times Y)$ and $\kappa X \times \kappa Y$ of $X \times Y$ are equal iff (1) $X$ or $Y$ is finite, or (2) $X$ and $Y$ are $H$-closed. In this note, we examine whether homeomorphism of these two extensions implies equality. We give a condition under which homeomorphism implies equality and an example to show that this relation does not hold in general. We also consider a number of related questions. We have been strongly motivated by the work of Gillman and Jerison [5] and McArthur [9], where analogous problems for the Stone-Čech compactification and the Hewitt realcompactification have been handled respectively.

2. Definitions and preliminaries. All spaces are assumed to be Hausdorff topological spaces. We let $R$ denote the space of real numbers with the usual topology and $N$ its subspace consisting of the positive integers. If the spaces $Y$ and $Z$ are homeomorphic, we write $Y \approx Z$. A space $Y$ is said to be an extension of the space $X$ if $c l_{Y} X=Y$. Two extensions $Y$ and $Z$ of $X$ are said to be equal, written $Y=Z$, if there is a homeomorphism $h: Y \rightarrow Z$ leaving $X$ pointwise fixed. Let $C(X)$ be the set of all real continuous functions on $X$ and let $C^{*}(X)=\{f \in C(X)$ : $f$ is bounded $\}$. A subset $A$ of $X$ is said to be $C^{*}$ embedded in $X$ if every function in $C^{*}(A)$ can be extended to a function in $C^{*}(X)$ [4]. A space $X$ is said to be $H$-closed if it is closed in every space in which it can be embedded. For information about $H$-closed spaces, the reader is referred to [8] and [10]. We denote the topological sum of the spaces $X$ and $Y$ by $X+Y$.

3. Unequal homeomorphic $H$-closed extensions. In [4, 6C.2], Gillman and Jerison point out that it can happen that a compactification $T$ of a Tychonoff space $X$ is homeomorphic with $\beta X$, the Stone-Čech compactification of $X$, without its being equal to $\beta X$. To see this, let $S=R \backslash N, X=S \backslash\{0\}$, and $T=\beta S$. Since $X$ is not $C^{*}$-embedded in $S$, it is not $C^{*}$-embedded in $T$. Thus $T \neq \beta X$ [4]. However, $S \approx X$ which implies that $\beta S$ or $T \approx \beta X$. Since a space is

Received by the editors December 18, 1974 and, in revised form, August 28, 1975. 
$C^{*}$-embedded in its Katětov extension, it follows that $U=\kappa S \approx \kappa X$ but $U \neq \kappa X$. Thus, an $H$-closed extension of a space $X$ may be homeomorphic to $\kappa X$ without its being equal to it.

In the above examples, that $T \neq \beta X$ and $U \neq \kappa X$ follows from the fact that $X$ is $C^{*}$-embedded in neither $T$ nor $U$. In the case of the Stone-Cech compactification, this reliance upon the $C^{*}$-embedding condition is critical: $\beta X$ is characterized by this property [4]. On the other hand, a space $X$ may have infinitely many unequal $H$-closed extensions in which it is $C^{*}$-embedded. This suggests that an example sharper than the one of the above paragraph may be given. Specifically, we exhibit a space $P$ having an $H$-closed extension $V$ with the properties that $P$ is $C^{*}$-embedded in $V, V \approx \kappa P$, but $V \neq \kappa P$.

If $N_{1}$ and $N_{2}$ are the subsets of $N$ consisting of the odd and even integers, split $N_{2}$ into disjoint infinite subsets $N_{3}$ and $N_{4}$. Let $P=N_{1}+\left(N_{3}+\beta N_{4}\right)$ and let $E=N_{1}+\beta N_{2}$. One can easily verify that $P$ is a dense $C^{*}$-embedded subset of $E$ and that $P \approx E$. If we let $V=\kappa E$, then $P$ clearly satisfies the first two of the desired properties. That $V \neq \kappa P$ follows from the fact that $V \backslash P$ is not discrete.

The above remarks do not rule out the possibility that $\kappa(X \times Y) \approx \kappa X \times \kappa Y$ implies that $\kappa(X \times Y)=\kappa X \times \kappa Y$. However, we shall presently produce an example showing that, in this situation also, homeomorphism need not imply equality. We shall make use of the following theorem of Lui:

3.1. If $T$ is an $H$-closed extension of a space $X$ with the properties

(1) all sets of the form $G \cup\{p\}$, where $G$ is open in $X$ and $p \in c l_{T} G \backslash X$, are open in $T$, and

(2) $X$ is open in $T$, then $T=\kappa X$. Conversely, $\kappa X$ is an $H$-closed extension of $X$ with these properties [8].

3.2. LEMMA. $\kappa(N \times \kappa N) \approx \kappa N$.

Proof. Since $N \times N \approx N, \kappa(N \times N) \approx \kappa N$ and it suffices to prove that $\kappa(N \times \kappa N)=\kappa(N \times N)$. For convenience, let $T=\kappa(N \times \kappa N)$ and we verify that (1) and (2) of 3.1 hold. If $G$ is open in $N \times N$ and $p \in c l_{T} G \backslash N \times N$, then either $p \in T \backslash N \times \kappa N$ or $p \in N \times \kappa N \backslash N \times N$. In the first situation, since $G$ is open in $N \times \kappa N$ and $p \in c_{T} G \backslash N \times \kappa N$, it follows that $G \cup\{p\}$ is open in $T$ by 3.1. On the other hand, if $p \in N \times \kappa N \backslash N \times N$, then $p$ has the form $(n, a)$ where $n \in N$ and $a \in \kappa N \backslash N$. If $Y=\{n\} \times \kappa N$, then $p \in c l_{Y}(G \cap(\{n\} \times N))$ and so again by (3.1), $[(G \cap(\{n\} \times N)) \cup\{p\}]$ is open in $Y$. It now follows easily that $G \cup\{p\}$ is open in $N \times \kappa N$ and hence in $T$.

3.3. LEMMA. If $L=N_{1}+\kappa N_{2}$, then $\kappa L \approx \kappa N\left(N_{1}\right.$ and $N_{2}$ are again the odd and even positive integers respectively).

Proof. Since $N_{1}$ and $\kappa N_{2}$ are disjoint closed subsets of $L$ whose union is $L$, $\kappa L \approx \kappa N_{1}+\kappa N_{2}$ by the lemma following Theorem 4.1 of [8]. Similarly, $\kappa N \approx$ $\kappa N_{1}+\kappa N_{2}$. 
3.4. EXAMPLE. $\kappa(L \times L)$ and $\kappa L \times \kappa L$ are homeomorphic but not equal. To show that they are homeomorphic, one would employ the previous two lemmas in an argument which is step by step analogous to the proof of Theorem 9 of [5]. That the extensions are not equal follows from Liu's theorem $(L \neq \kappa L)$.

4. Condition sufficient for homeomorphism to imply equality. Our next theorem will uitilize a result of Porter and Votaw: $X$ is not first countable at any point of $\kappa X \backslash X[11]$.

4.1. TheOREM. If $X$ and $Y$ are first countable, then $\kappa(X \times Y) \approx \kappa X \times \kappa Y$ implies that $\kappa(X \times Y)=\kappa X \times \kappa Y$.

Proof. Let $\tau: \kappa(X \times Y) \rightarrow \kappa X \times \kappa Y$ be a homeomorphism. Now $X \times Y$ is first countable, and hence each point of $X \times Y$ has a countable base of neighbourhoods in $\kappa(X \times Y)$. It follows that every point of $\tau(X \times Y)$ has a countable base in $\kappa X \times \kappa Y$. However, a point $(p, q)$ of $\kappa X \times \kappa Y$ has a countable base in $\kappa X \times \kappa Y$ iff $p$ and $q$ have countable bases in $\kappa X$ and $\kappa Y$ respectively. By Porter's result, $\tau(X \times Y) \subseteq X \times Y$. A similar argument with respect to $\tau^{-1}$ shows that $\tau^{-1}(X \times Y) \subseteq X \times Y$ and hence $X \times Y \subseteq \tau(X \times Y)$. From 3.1, it is easily seen that $\kappa X \times \kappa Y$ is the Katětov extension of $\tau(X \times Y)=X \times Y$ or $\kappa X \times \kappa Y=$ $\kappa(X \times Y)$.

5. Equality for infinite products. After determining when $\kappa(X \times Y)=$ $\kappa X \times \kappa Y$, Liu extended this result to infinite products as follows: $\kappa\left(\prod X_{\alpha}\right)=$ $\prod \kappa X_{\alpha}$ iff either (1) $\kappa X_{\alpha}=X_{\alpha}$ for all $\alpha$, or (2) there exists $X_{\beta}$ which is not $H$-closed, $X_{\alpha}$ is finite for $\alpha \neq \beta$, and all but finitely many $X_{\alpha}$ 's have only one point [8]. We claim that the proof of this theorem is incomplete since the necessary half is based on an assertion $\left(\kappa\left(X \times X_{\alpha_{0}}\right)=\kappa X \times \kappa X_{\alpha_{0}}\right)$ for which there is no justification. We have communicated with Professor Liu who has agreed with us on this point. We now complete the proof of this theorem.

Proof. Suppose $\kappa\left(\prod X_{\alpha}\right)=\prod \kappa X_{\alpha}$ so that there is a homeomorphism $\tau: \kappa\left(\prod X_{\alpha}\right) \rightarrow \prod \kappa X_{\alpha}$ leaving $\prod X_{\alpha}$ pointwise fixed. If (1) fails for say $\beta$, let $y \in \kappa X_{\beta} \backslash X_{\beta}$ and let $P=\left\{f \in \prod \kappa X_{\alpha}: f(\beta)=y\right\}$. Now $P$, being homeomorphic to $\prod_{\alpha \neq \beta} \kappa X_{\alpha}$, is $H$-closed, and $\tau^{-1}(P)$ is a subset of the discrete space $\kappa\left(\prod X_{\alpha}\right) \backslash \prod X_{\alpha}$. It follows that $P$ is finite and hence (2) holds.

6. The Fomin extension. Another well known $H$-closed extension of a space $X$ is the so-called Fomin extension, denoted by $\sigma X$; it is merely the underlying set of the Katětov extension with a coarser topology. This extension was introduced by Fomin [3] and subsequent work was done by Porter and Votaw [11] and Blazscyk [1]. In [7], Katětov showed that $\sigma X=\beta X$ iff the boundary of each open subset of $X$ is compact. In view of our earlier discussion, there are a number of obvious questions about $\sigma X$. We shall presently determine when $\sigma\left(\prod X_{\alpha}\right)=\prod \sigma\left(X_{\alpha}\right)$. 
6.1. THEOREM. If $X$ and $Y$ are spaces, then $\sigma(X \times Y)=\sigma X \times \sigma Y$ iff either (1) $X$ or $Y$ is finite or (2) $X=\sigma X$ and $Y=\sigma Y$.

Proof. The sufficiency of (2) is clear; that (1) is sufficient requires an argument analogous to the proof of the sufficiency of (a) of Theorem 4.1 of [8].

Suppose $\sigma(X \times Y)=\sigma X \times \sigma Y$. If $X$ and $Y$ are infinite, then either $X$ or $Y$ is not discrete: otherwise we would have $\beta(X \times Y)=\beta X \times \beta Y$ by Katětov's theorem, a fact incompatible with the well known result of Glicksberg [6]. Therefore, we may assume that one of the spaces, say $Y$, is not $H$-closed and that the other is not discrete. Choose $a \in \sigma Y \backslash Y$ (its elements are open ultrafilters) and an open set $V$ of $X$ with a point $p \in\left(c l_{X} V\right) \backslash V$. Denoting $\sigma X \times \sigma Y$ by $P$ and $X \times Y$ by $S$, since $P=\sigma(S)$ we can identify the points of $P \backslash S$ with open ultrafilters of $S$.

It follows that $(p, a) \in c l_{p}(V \times Y)=c l_{S}(V \times Y) \cup\{c \in P \backslash S: V \times Y \in c\}[1]$.

Now $(p, \boldsymbol{a}) \in V \times Y \cup\{c \in P \backslash S: V \times Y \in c\}=W$ which is open in $P$, but no basic open set about $(p, a)$ in the product topology of $\sigma X \times \sigma Y$ is contained in $W$, a contradiction.

6.2. THEOREM. If $\left\{X_{\alpha}\right\}$ is a collection of nonempty spaces, then $\sigma\left(\prod X_{\alpha}\right)=$ $\prod \sigma\left(X_{\alpha}\right)$ iff either (1) $\sigma X_{\alpha}=X_{\alpha}$ for all $\alpha$, or (2) there exists an $X_{\beta}$ which is not $H$-closed and $\prod_{\alpha \neq \beta} X_{\alpha}$ is finite.

Proof. That (1) is sufficient is obvious; the sufficiency of (2) follows from Lemma 6.1. Therefore, suppose $\sigma\left(\prod X_{\alpha}\right)=\prod \sigma\left(X_{\alpha}\right)$ and that (1) fails for say $\beta$. If $X_{\delta}$ were not discrete for some $\delta \neq \beta$, choose an open subset $V$ of $X_{\delta}$ with a nonempty boundary and let $g(\beta)=y \in \sigma\left(X_{\beta}\right) \backslash X_{\beta}, g(\delta)=p \in\left(c l_{X_{\delta}} V\right) \backslash V$, and $g(\alpha) \in X_{\alpha}$ for $\alpha \neq \beta, \delta$. With this $g \in \prod \sigma\left(X_{\alpha}\right)$, we can obtain a contradiction as we did in the proof of theorem 6.1. It follows that $X_{\alpha}$ is discrete for all $\alpha \neq \beta$.

Furthermore, if $X_{\beta}$ were not discrete, then by identical reasoning all other $X_{\alpha}$ 's are $H$-closed. From our hypothesis, it follows easily that $\sigma\left(X_{\beta} \times\right.$ $\left.\prod_{\alpha \neq \beta} X_{\alpha}\right)=\sigma\left(X_{\beta}\right) \times \prod_{\alpha \neq \beta} \sigma\left(X_{\alpha}\right)$ which implies that $\sigma\left(X_{\beta} \times \prod_{\alpha \neq \beta} X_{\alpha}\right)=\sigma\left(X_{\beta}\right) \times$ $\sigma\left(\prod_{\alpha \neq \beta} X_{\alpha}\right)$. By theorem $6.1, \prod_{\alpha \neq \beta} X_{\alpha}$ is finite.

Finally, we consider the case where $X_{\beta}$ is discrete. It follows that $\sigma\left(\prod X_{\alpha}\right)$ is compact and hence $\beta\left(\prod X_{\alpha}\right)=\prod \beta\left(X_{\alpha}\right)$. If $\prod_{\alpha \neq \beta} X_{a}$ were not finite, then from Glicksberg's Theorem [6], $\prod X_{\alpha}$ is pseudocompact which is impossible since $X_{\beta}$ is discrete.

The counterpart of Theorem 4.1 is easy to establish.

6.3. Theorem. If $X$ and $Y$ are first countable, then $\sigma(X \times Y) \approx \sigma X \times \sigma Y$ implies that $\sigma(X \times Y)=\sigma X \times \sigma Y$.

The proof is the same as the proof Theorem 4.1 except that the use of Theorem 3.1 is replaced by Theorem 1 in [1]. On the other hand we do not know whether homeomorphism of $\sigma(X \times Y)$ and $\sigma X \times \sigma Y$ implies equality. It 
is natural to attempt to show that there may be homeomorphism without equality by using the space $N_{1}+\sigma N_{2}=M$. However, it is easily verified that $\sigma(X \times Y)$ is compact iff (1) $X$ and $Y$ are discrete, (2) $X$ is finite and $\sigma Y$ is compact or vice-versa, or (3) $X$ is compact and $Y$ is compact. Since $\sigma(M \times M)$ is not compact, it is not homeomorphic to $\sigma(M) \times \sigma(M)$.

In [2], Flachsmeyer has shown that $\sigma X=\kappa X$ iff $\kappa X \backslash X$ is finite. In this situation also, we have been unable to determine whether homeomorphism implies equality.

7. ACKNOWLEDGEMENT. The author expresses his gratitude to the referee who made a number of valuable comments. Appreciation is also expressed to Professor Lester Gavurin, Chairman of the Mathematics Department at Brooklyn College, who obtained for the author a reduced teaching load during the spring semester 1974, during which time this article was written.

\section{REFERENCES}

1. A. Blaszcyk, On locally $H$-closed spaces and the Fomin $H$-closed extension, Coll. Math. 25, 241-253 (1972).

2. J. Flachsmeyer, Zur theorie der H-abgeschlossen erweiterungen, Math. Zeit. 94, 349-381 (1966).

3. S. Fomin, Extensions of topological spaces, Ann. of Math. 44 (2), 471-480 (1943).

4. L. Gillman and M. Jerison, Rings of continuous functions, Princeton, 1960.

5. Stone-Cech compactification of a product, Arch. Math. 10, 443-446 (1959).

6. I. Glicksberg, Stone-Cech compactifications of products, Trans. Am. Math. Soc. 90, 369-382 (1959).

7. M. Katětov, On H-closed extensions of topological spaces, Casopis Pest. Mat. 69, 17-32 (1947).

8. C. T. Liu, Absolutely ciosed spaces, Trans. Am. Math. Soc. 130, 86-104 (1968).

9. W. G. McArthur, A note on homeomorphic realcompactifications, Arch. Math. 22, 85-87 (1971).

10. J. Porter and J. Thomas, On H-closed and minimal Hausdorff spaces, Trans. Am. Math. Soc. 138, 159-170 (1969).

11. J. Porter and C. Votaw, H-closed extensions II, University of Kansas, 1973.

Brooklyn College of the City University of New York

UNIVERSIDAD Simón BolívaR 